\title{
- The Effects of Automatic Spelling Correction Software on Understanding and Comprehension in Compensated Dyslexia: Improved Recall Following Dictation
}

\author{
Lucy Hiscox, Erika Leonavičiūtė and Trevor Humby* \\ School of Psychology, Cardiff University, Park Place, Cardiff, CFIO 3AT, UK
}

\begin{abstract}
Dyslexia is associated with difficulties in language-specific skills such as spelling, writing and reading; the difficulty in acquiring literacy skills is not a result of low intelligence or the absence of learning opportunity, but these issues will persist throughout life and could affect long-term education. Writing is a complex process involving many different functions, integrated by the working memory system; people with dyslexia have a working memory deficit, which means that concentration on writing quality may be detrimental to understanding. We confirm impaired working memory in a sample of university students with (compensated) dyslexia, and using a within-subject design with three test conditions, we show that these participants demonstrated better understanding of a piece of text if they had used automatic spelling correction software during a dictation/transcription task. We hypothesize that the use of the autocorrecting software reduced demand on working memory, by allowing word writing to be more automatic, thus enabling better processing and understanding of the content of the transcriptions and improved recall. Long-term and regular use of autocorrecting assistive software should be beneficial for people with and without dyslexia and may improve confidence, written work, academic achievement and self-esteem, which are all affected in dyslexia. Copyright (C) 2014 John Wiley \& Sons, Ltd.
\end{abstract}

Keywords: compensated dyslexia; working memory; assistive software; autocorrection; writing; comprehension

\section{Key Messages}

- Working memory is a finite temporal storage of information, which integrates different ongoing behaviours and functions.

- In addition to the problems with language-specific skills shown by people with dyslexia, reduced working memory capacity is also found.

- If someone with dyslexia is concentrating on writing, and making sure that their spelling/ grammar is accurate, they might not fully appreciate the content of the information they are writing.

- Our results show that using autocorrecting assistive software during transcription increases the understanding of the content of the written information.

- We propose that using autocorrecting software during lectures/class will aid understanding of the presented material and may improve academic achievement.

*Correspondence to: Dr Trevor Humby, School of Psychology, Cardiff University, Tower Building, Park Place Cardiff CFIO 3AT, UK. E-mail: humbyt@cardiff.ac.uk 
- Our study investigated university students, that is, compensated dyslexia, but these findings may be extrapolated to pre-university students.

\section{INTRODUCTION}

Dyslexia is defined by the International Dyslexia Association (2008) as a languagebased disorder with difficulties in language-specific skills such as spelling, writing, pronouncing words and particularly reading; the difficulty in acquiring literacy skills is not a result of low intelligence or the absence of learning opportunity. Problems in the ability to spell and write persist throughout life (Bruck, 1993), affecting longterm education, and reading achievement will remain substantially lower than might be expected (Kirwan \& Leather, 20I I). However, within the adult dyslexia population, there are individuals where reading/writing achievements are sufficient to enable education at higher levels (Pennington et al., 1986). Referred to as 'compensated' dyslexia, these individuals, however, continue to show attenuated reading abilities (Ramus et al., 2003) despite employing different strategies to help them cope with more complex information and university-style teaching methods, such as lectures (Vasic et al., 2008).

There are many different forms of dyslexia, including phonological dyslexia, surface dyslexia and letter-by-letter reading (Ellis, 1984), demonstrating the different ways in which writing/language can be affected, that is, the structural/sound architecture of spoken or written words. People with dyslexia display high rates of spelling errors (Sterling et al., 1998), more word-related pauses, spelling-related editing (Wengelin, 2007), letter reversals (Brooks, Berninger, \& Abbott, 20II), reduced length of written composition (Sterling et al., 1998) and lower rates of task completion (Gregg, Coleman, Davis, \& Chalk, 2007). Although most of these findings originate from pre-higher-education samples, university students with dyslexia reported significantly more difficulties with spelling, note taking and expressing ideas compared with students without dyslexia, amongst other difficulties in a wide range of skills and academic tasks (Mortimore \& Crozier, 2007), suggesting a commonality of problems between compensated and noncompensated dyslexia, despite the greater demands of higher education.

Writing is a complex process involving many functions from the generation of the content to be inscribed to the motor control necessary to manipulate the pen or keyboard. The 'Simple Model of Writing' (Berninger \& Amtmann, 2003) proposed a triadic relationship between text generation, transcription and executive function, where transcription refers to handwriting/keyboarding and spelling ability and executive functions involve conscious attention, planning, reviewing, revising and strategies for self-regulation. Each of these processes requires the use of working memory, for integrating both low-level processing such as transcription and higher-level processing, including both executive functioning and text generation (Berninger \& Amtmann, 2003). Working memory, defined as a finite temporal storage of information where processing of surface features takes place until a goal-oriented task is completed (Goldman-Rakic, 1992), is a multicomponent system, comprising the phonological loop, the visual-spatial sketch pad and the modality-free central executive (Baddeley, 1992). Reduced working memory capacity is a consistent finding in dyslexia (Akerman, Dykman, \& Gardner, 1990; 
Smith-Spark \& Fisk, 2007; Beneventi, Tønnessen, Ersland, \& Hugdahl, 20I0; Helland \& Asbjornsen, 20 I0; Menghini, Finzi, Carlesimo, \& Vicari, 20I I). Furthermore, twin studies found that a verbal working memory deficit in young children was able to predict future dyslexia during the school years (Byrne et al., 2002). Although the majority of this work has been conducted in children, deficits in working memory performance have also been observed in older populations of compensated dyslexic individuals (Siegel, 1994; Brosnan et al., 2002; Miller-Shaul, 2005; Lindgrén \& Laine, 20I I).

It has been hypothesized that the different reading/writing processes compete for working memory (Berninger \& Swanson, 1994; Olive, 2004) and that individual differences in working memory capacity can constrain fluency of writing or transcription (Ransdell \& Levy, 1996) and may therefore underlie dyslexia (Swanson \& Ashbaker, 2000). Transcription fluency affects the quality of written work and the comprehension of transcribed material in both children (Olinghouse \& Graham, 2009) and adults (Olive, Alves, \& Castro, 2009). Difficulties with handwriting and assembling letters (orthographic motor integration) accounted for $67 \%$ of the variance in written expression, affecting the coherence and understanding of ideas in relation to the topic under study (Jones \& Christensen, 1999), and transcription fluency of lecture notes and text performance in a higher-education setting was the only significant predictor of note-taking quality, with the quality of notes the most important contributor to test performance, suggesting a linear relationship between written transcription fluency and recall test performance (Peverly, Ramaswamy, \& Brown, 2007).

Given the interactive nature of the processes involved in writing, the automaticity of transcription may be important in order to simultaneously engage in higherlevel processing necessary for reviewing and understanding written material. Therefore, theories of limited working memory capacity suggest that performance could be improved if one of the processes essential for writing was automatized (Peverly et al., 2007; Snowling \& Hulme, 20I I). Both basic instruction (Berninger et al., 1997) and compensatory computer-based methods such as spellcheckers (Graham \& Harris, 200I) have shown improvement in the quality of writing; however, spelling errors were not sufficiently recognized to consistently suggest possible correct spellings. Furthermore, students may not have recognized the correctly spelled word in the list of possibilities (MacArthur, Graham, Haynes, \& $\mathrm{De} \mathrm{La} \mathrm{Paz,} \mathrm{1996).} \mathrm{There} \mathrm{is} \mathrm{now} \mathrm{a} \mathrm{wide} \mathrm{range} \mathrm{of} \mathrm{assistive} \mathrm{technologies} \mathrm{to} \mathrm{help} \mathrm{with}$ reading/writing with text-to-speech, speech recognition and advanced spellcheckers (e.g. please visit the ICT section of the British Dyslexia Association for details: http:// bdatech.org). Although each of these interventions have had success in improving the quality of writing (e.g. Engstrom, 2005; Goldfus \& Gotesman, 2010), the effectiveness of these technologies in improving the ability of people to comprehend the information they have subsequently written has not been assessed.

Therefore, the current study aimed to investigate the consequences of writing automaticity and the effect it may have in the understanding of the content of written composition in compensated dyslexia, in a higher-education setting akin to a seminar or lecture. In addition to the quality and accuracy of transcription and recall of the material, we also assessed confidence in recall test performance. Anxiety and low self-confidence with written work have been found in people with dyslexia at both school and university, with university students feeling less competent both in their written work and in their academic achievements (Riddick, 
20/0). In a within-subject design, participants transcribed dictated text in Microsoft Word on a personal computer, under three different test conditions: Microsoft word spellchecker; Global AutoCorrect (GAC, LexAble Ltd, UK), an automatic and intelligent autocorrecting program designed to aid people with dyslexia; and a final condition with no computer/software assistance. It was hypothesized that the use of GAC autocorrecting software would reduce working memory demand, by allowing word writing to automatically flow, enabling better understanding of the content of the transcriptions and improved recall and confidence in recall $\sim 6 \mathrm{~h}$ later. Results showed that the autocorrecting software did not significantly improve recall for students without dyslexia but did significantly improve recall in students with compensated dyslexia.

\section{MATERIALS AND METHODS}

\section{Participants}

In total, 49 male and female undergraduate students from Cardiff University, recruited by opportunistic responses to posters displayed around the campus, participated in the study in return for course credit or a small financial gratuity (Table I). Students with dyslexia $(N=22)$ had all been assessed by educational psychologists and confirmed by the Student Support Centre of Cardiff University, which assists students with learning disabilities, and showed proof of their diagnosis by this unit at the initial experiment test session; subtypes of dyslexia were not investigated, and there was no evidence for co-morbid diagnoses. Nondyslexic participants $(N=27)$ self-reported no issues with their reading or writing abilities. All subjects were native English speakers; further information regarding educational achievement in English was also collected (Table I), showing that all had obtained high grades in secondary school exams for English; there was no difference in proportions of students with and without dyslexia formally studying English to a higher level (Table I). It should also be noted that the entrance requirements for most undergraduate courses at Cardiff are quite demanding; therefore, it could be assumed that participants with dyslexia were quite high functioning, that is, showing compensated dyslexia. All participants gave informed consent prior to inclusion in study, which was approved by the School of Psychology Ethics Committee, Cardiff University.

\section{Apparatus}

Working memory assessment

Forwards and reverse digit-span tests were used to assess working memory capacity (Spring, 1976; Vasic et al., 2008). Participants were presented with a number

Table I. Characteristics of the experimental and control groups

\begin{tabular}{lccccc}
\hline Participant group & $N$ & Male/female & $\begin{array}{c}\text { Age (years) } \\
\text { (mean } \pm \text { SEM) }\end{array}$ & $\begin{array}{c}\text { A-level } \\
\text { English exam }\end{array}$ & $\begin{array}{c}\text { GCSE English } \\
\text { grade }(\geq C)\end{array}$ \\
\hline Without dyslexia & 27 & $3 / 24$ & $19.19 \pm 0.17(18$ to 21$)$ & $5 / 27(18 \%)$ & $27 / 27$ \\
With dyslexia & 22 & $8 / 14$ & $21.40 \pm 0.53(18$ to 24$)$ & $3 / 22(14 \%)$ & $22 / 22$ \\
\hline
\end{tabular}


of series of digits, ranging in length from four to nine (forwards) or four to six (reverse), with the presentation of each sequence terminated by a sharp tap to a desk. Three different randomly generated sequences of digits were presented for each length and for each test. On hearing the 'tap', the participants were to record the digits they had heard, in the order with which they had been presented. For consistency, the digit sequences and 'taps' were recorded on a CD, and participants wrote their answers to a Microsoft Word document, open on the computer in front of them.

\section{Transcription task}

Three excerpts from crime fiction novels of equal length and complexity were used in the different transcription sessions (Table 2); no identification of the source material was available to the participants. A pilot study showed that few undergraduate students from Cardiff University had heard of the authors of the books from which the transcripts had been taken, and participants, when asked on completion of the experiment, could not identify the source material used. Reading of the different excerpts was recorded for consistency of presentation, and pilot work showed that a reading speed of $\sim 45$ words per minute produced the most reliable presentation for transcription. We also found that reading the words in three to eight word clusters, dependant on word length, with brief periods of silence ( $\mathrm{I}-2 \mathrm{~s}$ ) separating each word cluster also enabled more accurate transcription. The length of silences was also determined during pilot studies, with the aim that participants would only have brief pauses between sets of words. Words deemed too unusual were replaced with a more familiar alternative, although all nouns were excluded from the final analysis of individual transcriptions made by the participants (Table 2). During the transcription phase of the experiment, all key presses and mouse movements at the computer made by the participants were recorded. These actions were incorporated into an adapted version of the GAC assistive software (LexAble Ltd), which was employed as one of the test conditions, although the autocorrect function was disabled for two of the test conditions (Table 3). Key presses and mouse movements were time stamped and

Table 2. Characteristics of the texts used in the transcription/recall task

\begin{tabular}{lccc}
\hline & Frederick Forsyth, & lan Rankin, & Dick Francis, \\
& The Fourth Protocol & The Impossible Dead & Silks \\
\hline Words & 627 & 631 & 582 \\
Letters & 2835 & 2916 & 2713 \\
Nouns removed from analysis & 31 & 43 & 26 \\
Mean transcription time & $19: 55$ & $20: 10$ & $20: 15$ \\
\hline
\end{tabular}

Table 3. Outline of the different transcription conditions employed

\begin{tabular}{lcccc}
\hline $\begin{array}{l}\text { Test } \\
\text { condition }\end{array}$ & $\begin{array}{c}\text { Global AutoCorrect } \\
\text { (LexAble Ltd) }\end{array}$ & $\begin{array}{c}\text { Microsoft Word } \\
\text { autocorrect }\end{array}$ & $\begin{array}{c}\text { Microsoft Word } \\
\text { manual spellcheck }\end{array}$ & $\begin{array}{c}\text { Instructed to be as } \\
\text { accurate as possible }\end{array}$ \\
\hline 1 & Enabled & Disabled & Enabled & Yes \\
2 & Disabled & Disabled & Enabled & Yes \\
3 & Disabled & Disabled & Enabled & No \\
\hline
\end{tabular}


saved to file for later analysis. The parameters of interest were the number of letters (characters typed), number of correct words, number of incorrect words, number of backspaces (character deletions), the number of manual spellchecks and the rate of writing determined as the number of words typed per minute. Importantly, the number of words autocorrected by the GAC program (for condition I only) was also determined, although we were also able to determine the amount of words that would have been corrected in conditions 2 and 3. Accuracy of the final transcription was determined by using the Microsoft Word compare documents function to delineate the differences between the original text and the text produced by each participant. Differences were then classified as follows as missing words, transposed words, additional words, spelling mistakes and text revisions (insertions or deletions). These errors were totalled, and a typing accuracy score was expressed as the ratio of total errors against the number of words for each text. Punctuation and nouns were excluded from all analyses.

\section{Recall test}

To determine the level of knowledge and understanding of the different dictated texts, questionnaires specific for each piece of text were designed. Each questionnaire was composed of I 5 questions and would take about 15 min to complete: six questions were multiple choice (MCQ) and assessed factual information, and the remaining nine were open-ended questions assessing comprehension and understanding (CUQ). Of the MCQs, two contained two possible answers (e.g. 'What had been the verdict of the jury?', guilty/not guilty), with the other four giving an option of three possible answers (e.g. 'What song did a character start humming?', options given). For MCQs, the definitive answer had been present in the dictated text, whereas for the CUQs, the answer would have to be derived from the context, or descriptions made in the text; however, there could still only be a single correct answer (e.g. 'What season was the piece of writing set in?'). Therefore, both question types were marked as either correct or incorrect; there was no scale of assessment. For each question, participants were asked to rate how confident they were with their answer, using a scale ranging from $0 \%$ (not at all confident) to $100 \%$ (extremely confident). The individual questionnaires were sent by email, approximately $6 \mathrm{~h}$ after completion of the transcription, and each participant was asked to return a completed set of questions within $24 \mathrm{~h}$.

\section{General Procedure}

Each participant took part in three separate sessions, each taking place a week apart. Each session involved listening to one of the pieces of text (Table 2) and making a transcription by typing into a Microsoft Word file on a computer. The transcription conditions and text were randomized for each session, such that each participant experienced each of the three test conditions (Table 3) and transcribed each of the three texts, although the order of presentation of each was counterbalanced between participants, such that members of both the dyslexic and nondyslexic groups could receive each text in conjunction with the different experimental conditions. Participants were told that they should aim to write down as much of the text as possible, carry on typing even if they had missed some excerpts and produce an as 'accurate as possible' transcription of the dictated text. They were also prewarned that they will be asked about the content of the 
dictated material. In condition I, some spelling mistakes would be automatically corrected during transcription, by the GAC spelling correction software (LexAble Ltd), running a standard default configuration of words to be autocorrected. In condition 2, spelling errors were not autocorrected; however, in both conditions I and 2, participants were instructed to make typing accuracy a priority. In condition 3, participants were instructed not to worry about spelling, that it was not necessary to correct mistakes and that they should instead focus on the content of the dictation. The Microsoft Word autocorrect function was disabled in all conditions. Therefore, condition 2 could be interpreted as a 'normal' condition, where working memory demands on typing accurately could interfere with attention to the content of the dictation, whereas in condition I, where the autocorrecting software was present to help, and condition 3 , where less demands were placed on accurate transcription, it was expected that the participants could attend to the dictated information better.

On average, between 6 and 12 people were assessed at any one time, a mix of participants with and without dyslexia, each sitting at a computer. Within each test session, only a single text was presented, played from a CD through loud speakers, but the participants experienced different test conditions. Each participant received information that was specific to their assigned test condition, and the rules were also carefully explained before the dictation was started, with the main overriding rule to all participants that they should transcribe as best they could to the speed of dictation. Overall, each dictation session took about 20 min to complete, although the additional collection of consent, personal information and the digit-span test meant that the first session was generally 30 min long. On completion, the key-logging data were saved, and the participants debriefed and asked to return for the next session. The questionnaires to assess learning were sent out $\sim 6 \mathrm{~h}$ after each dictation session.

\section{Data Analysis and Statistics}

All statistical analyses were carried out using SPSS v.20 for windows (IBM, Armonk, NY, USA). Percentage differences (response accuracy and omitted trials) were subject to an arcsine transformation prior to ANOVA in order to normalize the data (skewed data can result from high levels of performance shown by subjects relative to the 100\% maximum, Hogg and Craig, 1995). Digit-span performance was also arcsine transformed and then assessed by separate analyses for the forwards and reverse conditions with ANOVAs with a between-subject factor of group (dyslexia or nondyslexia) and a within-subject factor of sequence length (four to nine and four to six for forwards and reverse sequences, respectively). Recall test questionnaire data, percentage correct and confidence rating, were analysed using ANOVA with a between-subject factor of group and within-subject factors of condition (I, 2, 3) and question type (MCQ or CUQ). Accuracy of transcription and typing parameters were analysed by separate ANOVAs with a between-subject factor of group and within-subject factors of condition. Post hoc pairwise comparisons were performed using the Bonferroni test where appropriate, with alpha values of $<0.05$ regarded as significant. All data were assessed for normality prior to analysis, and Greenhouse-Geisser degrees of freedom corrections were applied as necessary to repeated-measures factors in relevant ANOVAs (nominal values for the relevant degrees of freedom are reported). 


\section{RESULTS}

Overall, 49 participants were recruited for the study, 22 with a diagnosed form of dyslexia and 27 without. Two participants (one member of each test group) did not complete all three test sessions, and therefore, group averages were substituted for the missing values. Although there was a slight difference in age, with the mean age of the dyslexic participants being older than that of the nondyslexic control subjects (Table I), the age ranges were similar, and all participants were undergraduate students at Cardiff University (Table I). All participants had English as a first language and had all achieved a grade of C or above in the GCSE English exam. Not many of these participants had taken English as a subject for further study, at A level, which may not be surprising as the majority of participants were undergraduate students studying psychology. However, all showed a good level of education and achievement in English examinations and had achieved good grades to enrol at the university. Therefore, the participants in this study were all quite highachieving students that had performed well at high school (and above) levels of education.

\section{Assessment of working memory}

As expected, increasing the length of each sequence of numbers made it more difficult for the participants to correctly recall the digits (Figure I, main effect of sequence length, $F_{5,235}=160.25, p<0.001$, and $F_{2,94}=59.53, p<0.001$, for forwards and reverse digit-span tests, respectively). This was more apparent in the reverse digit-span test, where recall of the correct sequence of digits was affected, even with only five numbers present. Participants with dyslexia showed significantly attenuated performance in both the forwards (main effect of group, $F_{I, 47}=|I .7|, p<0.00 I$ ) and reverse (main effect of group, $F_{I, 46}=16.17$, $p<0.00 \mathrm{I})$ conditions. A significant interaction between group and sequence length showed that these effects were most prominent when sequence length was greater than six digits for the forwards test $\left(F_{5,235}=4.54, p<0.00 \mathrm{I}\right)$, whereas a lack of significant interaction in the reverse test $\left(F_{2,94}=1.80\right.$, n.s. $)$ suggests a larger impairment in recall in this test by the participants with dyslexia. Overall, these data are consistent with a reduced working memory capacity in the participants with compensated dyslexia.
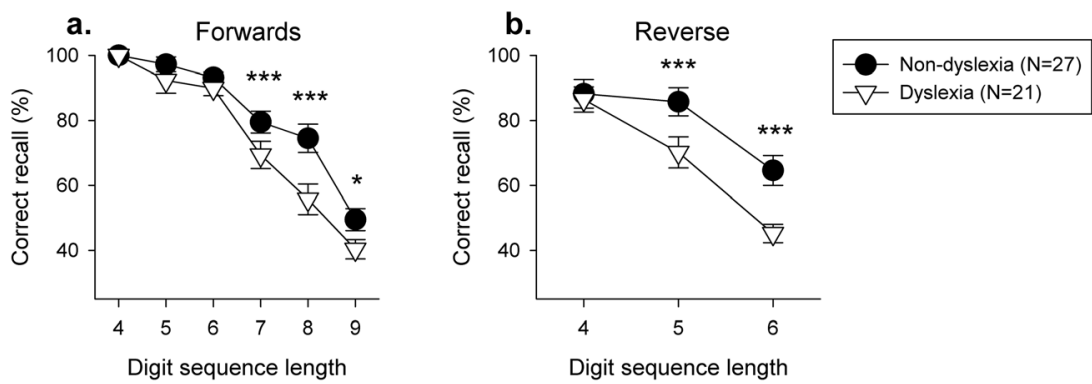

Figure I. Assessment of working memory using forward (a) and reverse (b) digit span tests. Data shows mean + / - SE. 
Text recall test

Following each dictation session, participants were sent a questionnaire related to the specific text they had heard and were also asked to report their confidence with their answers. All participants answered each of the questions and returned the completed questionnaires within $24 \mathrm{~h}$ of receipt. All participants found the CUQ more difficult than the MCQ (Figure 2a, main effect of question type, $\left.F_{1,47}=240.58, p<0.00 I\right)$. There were no overall significant differences between the participants with and without dyslexia (main effect of group, $F_{1,47}=2.95$, n.s.) or between the transcription conditions (main effect of condition, $F_{2,94}=1.48$, n.s.), but there was a significant interaction between these factors $\left(F_{2,94}=3.83\right.$, $p<0.025)$. Pairwise comparisons investigating the participants with dyslexia only showed a significant main effect of condition $\left(F_{2,42}=3.34, p<0.05\right)$, which was due to better recall between test conditions I and $2(p<0.033)$. This effect was more pronounced for the CUQs than the MCQs as shown by a significant interaction between condition and question type $\left(F_{2,42}=3.68, p<0.05\right)$. No such significant effects were found for participants without dyslexia (main effect of condition, $F_{2,52}=1.84$, n.s., and interaction between condition and question type, $F_{2,52}=0.54$, n.s.). Thus, these findings would suggest that the participants with dyslexia demonstrated improved recall following transcription in the situation where the GAC autocorrecting software was present (condition I); this was most evident in comparison with a 'normal' set-up (condition 2). Therefore, the use of autocorrecting assistive software improved recall for the participants with dyslexia, but not those without dyslexia. There was a significant effect of condition $\left(F_{2,42}=5.89, p<0.01\right)$ if the answers to CUQs for the participants with dyslexia were analysed alone, with pairwise comparisons showing that recall in test condition 2 was significantly different from both the other conditions $(p=0.024$ and $p=0.002$, for comparisons with conditions I and 3 , respectively); there was no significant difference between test conditions I and $3(p=0.58)$. The equivalent analysis for nondyslexic participants was not significant (main effect of condition, $F_{2,52}=2.95$, n.s.), neither were the analyses for MCQs (main effect of condition, $F_{2,42}=2.94$ and $F_{2,52}=0.31$, both n.s. for dyslexic and nondyslexic participants, respectively). Therefore, the attenuated recall shown by the dyslexic participants was mainly driven by the CUQs, and the assimilation and comprehension of the dictations were most affected in the test condition where the participants had to perform manual corrections (condition 2); the presence of the GAC automatic spelling correction
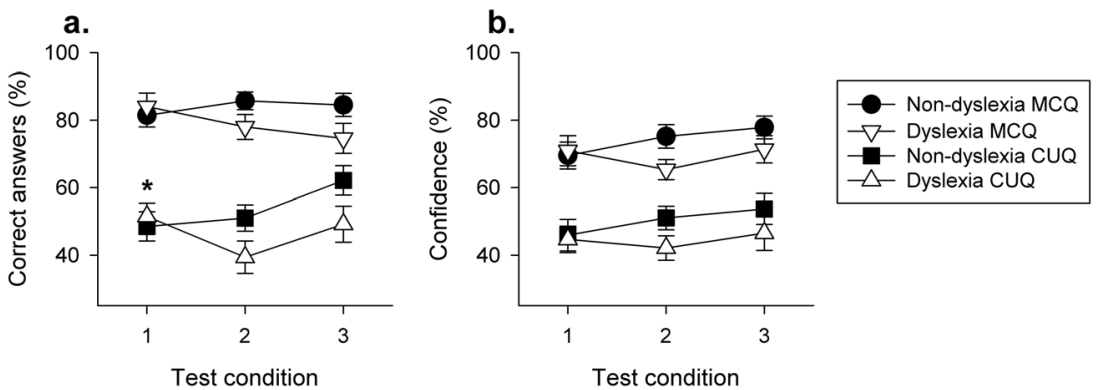

Figure 2. Text recall test (a) and confidence in answering (b). MCQ: multiple choice questions, CUQ: comprehension/understanding questions. See Table 3 for definition of test conditions. Data shows mean +/- SE. 
software during transcription (condition I) increased correct answering to a level beyond that of simply not having to make corrections (condition 3).

All participants were more confident with their answers to the MCQ than to the CUQ (Figure $2 b$, main effect of question type, $F_{I, 47}=262.75, p<0.00 \mathrm{I}$ ), suggesting that they found the CUQs more difficult than the MCQs, as expected. Levels of confidence in answering the questions did not differ between participants with and without dyslexia (main effect of group, $F_{I, 47}=2.99$, n.s.), and the condition under which the text transcription was made did not alter confidence in answering the questions (main effect of condition, $F_{2,94}=2.38$, n.s.). There was no significant interaction between these factors $\left(F_{2,94}=2.64\right.$, n.s. $)$. Therefore, the transcription conditions did not affect confidence in answering the questions, and participants with and without dyslexia showed equivalent levels of confidence in their answers. Overall, the pattern of results would suggest that all participants found it more difficult to answer questions correctly and were less confident when they had to concentrate on the transcription of the dictation (condition 2); this was especially prominent for CUQ. Participants with dyslexia showed a significant improvement when they were able to use the automatic spelling correction software to help their typing (condition I), this effect was present for both MCQ and CUQ, but only significantly so for CUQs.

\section{Accuracy of transcription and typing}

A key consideration related to the recall data was that the conditions imposed during each transcription session impacted on the accuracy and quality of the typing performed by the participants. Transcription accuracy was determined by comparing the final documents produced by the participants with the original dictated texts and counting differences in inserted or deleted characters, additional or missing words, word transpositions and spelling mistakes. An accuracy score was determined for each participant, for each test condition, by calculating the ratio of the total errors made to the number of words in each text.

There was no significant difference in the overall number of words typed by each group of participants $\left(F_{1,47}=1.86, p=0.18\right)$, with participants with dyslexia typing $569.35 \pm 2.52$ and nondyslexic students typing $574.67 \pm 2.86$ words averaged across each test condition. As noted in the Materials and Methods section, the transcripts were presented at a rate of $\sim 45$ words per minute with the words presented in clusters of between three and eight words each, with each word cluster separated by a gap of $\mathrm{I}-2 \mathrm{~s}$. Under these conditions, there was no significant difference in typing speed between students with and without dyslexia $\left(F_{1,47}=2.79, p=0.15\right)$, with rates of typing easily within the speed of transcription presentation speed: $28.79 \pm 0.15$ and $28.24 \pm 0.19$ words per minute averaged across all test conditions for dyslexic and nondyslexic participants, respectively. Participants with dyslexia made many more errors than the nondyslexic participants (Figure 3a, main effect of group, $F_{1,47}=31.89, p<0.0001$ ), and, as expected, all participants made more errors in condition 3 , where they did not have to concentrate on typing accurately, than the other test conditions (main effect of condition, $\left.F_{2,94}=10.59, p<0.001\right)$. A near-significant interaction between these factors $\left(F_{2,94}=10.59, p=0.058\right)$ suggested a trend for participants with dyslexia to be much more affected than nondyslexic participants in test condition 3 . All participants were generally very accurate in their transcription, with $>70 \%$ 

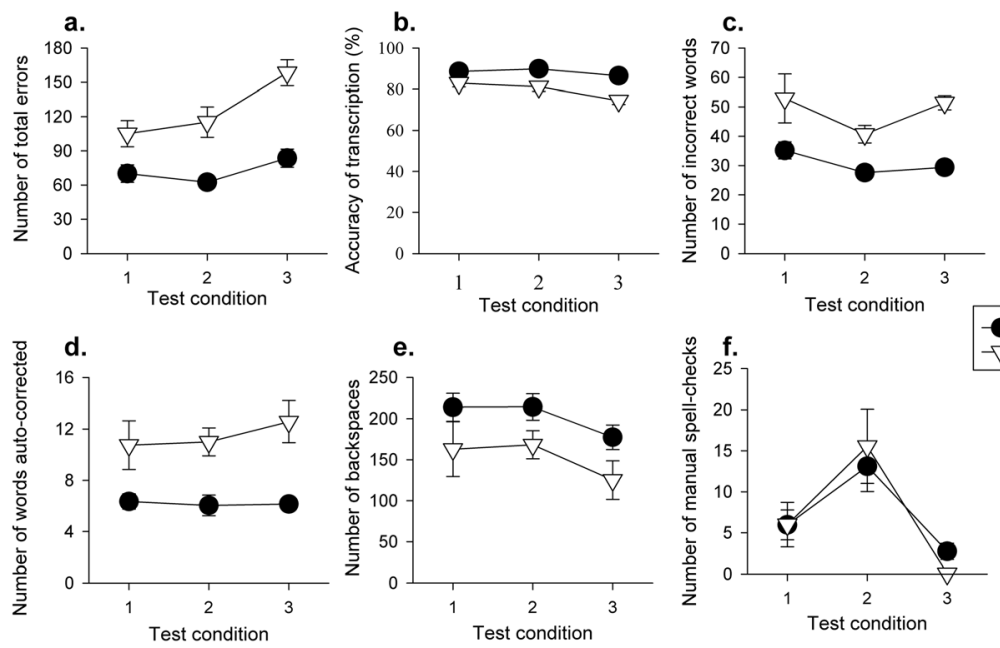

Non-dyslexia $(\mathrm{N}=27)$ Dyslexia $(\mathrm{N}=22)$

Figure 3. Accuracy of transcription and typing was assessed in terms of total errors made (a) and writing accuracy (b). The number of incorrect words typed (c) and autocorrected (d), and the number of deletions (e) and spell-checks ( $f$ ) were also analysed. See text for definitions of parameters and Table 3 for description of test conditions. Data shows mean +/- SE.

similarity to the original text (Figure 3b); however, participants with dyslexia were less accurate on average (main effect of group, $F_{1,47}=31.25, p<0.000 I$ ). Significant effects of condition $\left(F_{2,94}=10.79, p<0.001\right)$ and group * condition $\left(F_{2,94}=3.09, p<0.05\right)$ showed that transcription accuracy was decreased in condition 3 and that participants with dyslexia were far less accurate in this situation.

During transcription, mouse movements and keyboard presses were recorded so that online typing could be analysed. Comparing key presses that had been matched to the words in each text showed that participants with dyslexia typed more incorrect words then those without dyslexia (Figure 3c, main effect of group, $\left.F_{1,47}=17.48, p<0.0 \mathrm{I}\right)$ and had many more words autocorrected (Figure 3d, main effect of group, $F_{1,47}=27.47, p<0.00$ I). NB: The autocorrection function was only present for test condition I, but for analysis purposes, comparisons have been made using the number of autocorrections that would have been made if the function had been enabled in test conditions 2 and 3. In fact, an equivalent number of words would have been autocorrected in each test condition (main effect of condition, $F_{2,94}=0.68$, n.s.). Consistent with these findings, participants with dyslexia made fewer backspaces (single-character deletions) than nondyslexic participants (Figure $3 e$, main effect of group, $F_{1,47}=9.18, p<0.01$ ), and all participants showed fewer corrections in test condition 3, where they had been asked to be less concerned with the errors that they had made (main effect of condition, $F_{2,94}=7.83, p<0.01$ ). Likewise, there were significant differences between the different test conditions in terms of the manual spellchecker function of Microsoft Word (Figure 3f, main effect of condition, $F_{2,94}=22.0 \mathrm{l}$, $p<0.00 \mathrm{I})$. Usage was greatest in test condition 2 , where the participants were asked to produce an accurate transcription of the dictation but did not have the support of the autocorrection software, and lowest in test condition 3, where they had been asked not to correct their typing, pairwise comparison between conditions 2 and 3: $p=0.00 \mathrm{l}$. It was also noticeable that there was a significant reduction in the use of the manual spellchecker between test conditions $I$ and 2 , 
$p=0.00 \mathrm{I}$ for pairwise comparison, suggesting that the autocorrection software was functioning to correct words before the participant noticed. There was an equivalent use of the manual spellchecker between participants with and without dyslexia (main effect of group, $F_{1,47}=0.96$, n.s.).

Overall, these data show that participants with dyslexia made many more errors during transcription and produced less accurate renditions of each text. The imposition of the different test conditions (Table 3) was effective, in that the autocorrecting assistive software was successful in correcting typing errors online, and all participants responded to the relaxation in accuracy asked for in test condition 3, as shown by increased errors, reduced use of the Microsoft Word spellchecker and reduced accuracy of the final transcription.

\section{DISCUSSION}

This study aimed to investigate the consequences of writing automaticity and the effect it may have in the recall and understanding of the content of written composition in participants with compensated dyslexia, in comparison with nondyslexic participants. The task involved transcribing pieces of dictated text, similar to recording the content of a seminar or lecture. Results demonstrated that the presence of a piece of assistive software improved comprehension and understanding of the content of transcribed text by the participants with dyslexia only, shown by improved performance in a test of recall, predominantly driven by improvements in the extrapolation from, and the comprehension of the material (CUQs) rather than just recounting basic factual information (MCQs). There was a tendency for participants with dyslexia to be less confident with their answers during the recall test, but all participants were more confident at answering MCQs than the more in-depth comprehension questions. During transcription, participants with dyslexia made many more typing errors, were less accurate in their typing and had many more spelling errors corrected by the assistive software, although typing speed did not differ. Therefore, the overall pattern of results would suggest that use of assistive software during typing may be beneficial for people with dyslexia, not only in terms of the accuracy and clarity of their written text but also in terms of giving them a more in-depth understanding and synthesis of the content of the material they are dealing with.

We utilized a novel design, involving transcription into a Microsoft Word document of pieces of dictated text, and a subsequent test of recall about the content. Although students with compensated dyslexia have overcome a number of disadvantages to reach university, they may still exhibit difficulties with reading (refer to later discussion), which could impact upon studies involving writing, especially under time restraints. However, in the main part of the current experiment, the dictation/transcription test, the participants were not required to read text to complete the task; therefore, any differences in reading speed would not impact on this phase of the experiment. Likewise, although the participants had to read the response questionnaires sent out after the transcription task, these could be answered at the participant's own speed, and therefore, reading speed/ability would also not be an issue for this part of the experiment. Participants were always asked to be as accurate as possible in their transcription, demonstrated by the high levels of accuracy in comparison with the original texts and to also 
focus on the content of the material. Using a within-subjects design, participants conducted three separate transcriptions under different writing conditions. Effects of the different transcription conditions were greatest for the CUQs, although it was noticeable that students with dyslexia did also show lower levels of correct answers in the MCQs in comparison with nondyslexic participants. Students with dyslexia performed worst in the recall test following transcription in condition 2 (no autocorrection, but mistakes needed to be corrected), which could be interpreted as the most 'normal' situation and where working memory demands on typing accuracy and error correction were most likely to interfere with attention to the content of the dictation, consistent with previous studies (e.g. Peverly et al., 2007; Snowling \& Hulme, 20I I). When the need for spelling correction during transcription was removed by the GAC (www.lexable.com) automatic spelling correction software (condition I), the level of understanding and therefore the number of correct answers made by students with dyslexia significantly increased, even though the number typing errors made was equivalent to the amount made in the other test conditions. Thus, we would conclude that when the demands on working memory, in terms of correcting typing, were removed, understanding and comprehension of the dictated texts was increased.

Writing is an integrative activity, with different processes, such as text generation, transcription, attention, planning, reviewing and revising, which includes rereading the typed or written text, and strategies for self-regulation with the different systems each requiring working memory; overload or impairment of working memory may lead to writing difficulties (Ransdell \& Levy, 1996; Berninger \& Amtmann, 2003; Olive, 2004). Reduced working memory capacity is a consistent finding in dyslexia (Akerman et al., 1990; Smith-Spark \& Fisk, 2007; Beneventi et al., 20 I0; Helland \& Asbjornsen, 20 I0; Menghini et al., 20l I), including in older populations of compensated dyslexic individuals (Siegel, 1994; Miller-Shaul, 2005; Lindgrén \& Laine, 20II) replicated in the current work with undergraduate students using forwards and reverse digit-span tests (Spring, 1976; Brosnan et al., 2002; Vasic et al., 2008). Correctly encoding written words and amending spelling problems take cognitive capacity from other processes such as vocabulary choice and sentence structuring, thus impeding comprehension (Olive \& Kellogg, 2002; Wengelin, 2007). Thus, we would hypothesize that when the demands of correcting typing during the transcription stage of the current experiment were removed by the autocorrecting software (condition I), the participants with dyslexia were better able to attend to the content of the dictations and therefore showed improved recall subsequently. This is consistent with previous studies, using different methods, where transcription fluency or quality of note taking has been related to comprehension and understanding (Jones \& Christensen, 1999; Peverly et al., 2007; Olinghouse \& Graham, 2009; Olive et al., 2009; Snowling \& Hulme, 20I I).

Global AutoCorrect, the assistive software program used in the current study, is an intelligent, automatic-correcting software program that works in the background, performing more advanced functions than the in-built Microsoft Word autocorrection system. GAC can be individually customized by addition of words to be corrected, but in the current experiment, only a standard configuration with common typing errors was used. However, despite this functionality, the effect of GAC on recall, and increasing confidence of answers, was only present in students with dyslexia and was not found at all for the nondyslexic participants. For all participants, transcription with the assistive software was only conducted with a 
single 20-min session, with no practice beforehand; therefore, neither group would have been able to experience and gain confidence with the full capabilities of the program. The students with dyslexia may have more experience of using assistive software, hence would be more confident in its capabilities and so would show a bigger benefit. The lack of effect in participants without dyslexia may be due to a number of different reasons including 'normal' working memory enabling better coping with the demands of the task, more confidence in their own writing abilities and more robust strategies for typing and understanding. Moreover, students without dyslexia are less likely to have experience with this type of software; most of these technologies are targeted to dyslexia, and so they will be less confident in their use. Therefore, larger effects on understanding, comprehension and confidence in answering could be obtained in future studies, with GAC or other automatic spelling correction software, if the participants were to be given more training with the software and personalized the autocorrection list. Longterm and regular use of these types of programs should be beneficial for people with and without dyslexia and may improve confidence ratings and complexity in written work, academic achievement and self-esteem, which are all reduced in dyslexia (Sterling et al., 1998; Gregg et al., 2007; Riddick, 20I0).

The current study used high-functioning undergraduate university students who had achieved high secondary school grades for English, categorized as compensated dyslexia. Although measures of success in examinations were used as a proxy for reading ability, examination performance is not necessarily a direct measure of reading ability. Although it should be noted that attaining high grades in exams and attending university would suggest that these students with dyslexia are more likely to read more than people with dyslexia that are not in further education. However, as noted, the reading ability (and possible inclination to read) of undergraduate students with dyslexia may still not be equivalent to students without dyslexia. Linked with this is the possibility that students without dyslexia were more likely to have read the novels from which the dictations had been taken from. However, enquiries of the student population, and the participants following completion of the study, suggested that the novels (and authors) selected were unknown. It may be possible that the intervention used was not as beneficial to students with compensated dyslexia as it might be for other people with dyslexia, as they may have already learnt to compensate for reading/writing difficulties throughout their educational experience. There is evidence to suggest that people with dyslexia increasingly recruit additional speech-related prefrontal cortical regions of the brain over time, in order to compensate for the dysfunction (Vasic et al., 2008); therefore, larger effects may be found using children of school age, who function lower academically and who may not be fully compensating for their impairment. A further way in which a larger effect could be found would be to use self-generated text rather than transcription, as writing is more fluent if following dictation (Dockrell, 2009). Thus, the task could be made more difficult if the participants had to generate their own text composition, increasing working memory demands and therefore the possible effectiveness of the autocorrecting software. However, the experiment would be less controlled, and results maybe harder to interpret. GAC is, of course, not the only spelling correction software program available; there is now a wide range of assistive technologies to help with reading/writing with text-to-speech, speech recognition and advanced spellcheckers (e.g. please visit the ICT section of the British Dyslexia Association for details: http://bdatech.org). 
For the current experiment, GAC was chosen because it makes automatic corrections with higher frequency and greater accuracy than alternative products. However, to our knowledge, there are no studies that have specifically investigated the links between transcription, working memory and comprehension using other software programs that automatically correct typing/writing, but we would expect similar beneficial effects to those found in the current study.

\section{CONCLUSIONS}

The aim of the current study was to investigate the relationship on working memory capacity and accuracy of recalling information presented during a text transcription task. Writing is a complex process involving many different functions, integrated by the working memory system; people with dyslexia have a working memory deficit, which may represent the underlying aetiology of this disorder, which means that focus on writing quality may be detrimental to understanding and comprehension. We hypothesize that use of automatic autocorrecting software by students with dyslexia allowed more working memory capacity to be devoted to the content of dictations and therefore improved assimilation of the information and later recall of the content. Therefore, regular use of this type of software improved writing and comprehension and may engender more confidence in people with dyslexia, which may impact on self-esteem, academic achievement and future career choices (Jordan, 2002).

\section{ACKNOWLEDGEMENTS}

We would like to thank the undergraduate students of Cardiff University who participated in the study and Neil Cottrell of LexAble Ltd (for further information, visit www.lexable. com, e-mail: hello@lexable.com) for expert assistance with all things to do with the recording of data during the transcription sessions and for permitting access and use of his company's software: Global AutoCorrect.

\section{DISCLOSURE/CONFLICT OF INTEREST}

The authors declare no competing financial interests.

\section{REFERENCES}

Akerman, P. T., Dykman, R. A., \& Gardner, M. Y. (1990). Counting rate, naming rate, phonological sensitivity, and memory span: Major factors in dyslexia. Journal of Learning Disabilities, 5, 325-327.

Baddeley, A. (1992). Working memory: The interface between memory and cognition. Journal of Cognitive Neuroscience, 4, 28I-288.

Beneventi, H., Tønnessen, F. E., Ersland, L., \& Hugdahl, K. (2010). Working memory deficit in dyslexia: Behavioral and FMRI evidence. International Journal of Neuroscience, I 20, 5I-59.

Berninger, V. W., \& Amtmann, D. (2003). Preventing written expression disabilities through early and continuing assessment and intervention for handwriting and/or spelling problems: Research into practice. 
In H. L. Swanson, K. R. Harris, \& S. Graham (Eds.), Handbook of learning disabilities (pp. 345-363). New York: Guilford Press.

Berninger, V. W., \& Swanson, H. L. (1994). Modifying Hayes and Flower's model of skilled writing. In E. Butterfield (Ed.), Children's writing; toward a process theory of development of skilled writing (pp. 57-8I). Greenwich, CT: JAI Press.

Berninger, V. W., Vaughan, K. B., Graham, S., Abbott, R. D., Abbott, S. P., Rogan, L. W., .. Brooks, A. (1997). Treatment of handwriting fluency problems in beginning writing: Transfer from handwriting to composition. Journal of Educational Psychology, 89, 652-666.

Brooks, A. D., Berninger, V. W., \& Abbott, R. D. (20I I). Letter naming and letter writing reversals in children with dyslexia: Momentary inefficiency in the phonological and orthographic loops of working memory. Developmental Neuropsychology, 35, 847-868.

Brosnan, M., Demetre, J., Hamill, S., Robson, K., Shepherd, H., \& Cody, G. (2002). Executive functioning in adults and children with developmental dyslexia. Neuropsychologia, 40, 2I44-2I55.

Bruck, M. (1993). Component spelling skills of college students with childhood diagnoses of dyslexia. Learning Disability Quarterly, 16, 171-184.

Byrne, B., Delaland, C., Fielding-Barnsley, R., Quain, P., Samuelsson, S., Hoien, T., ... Corley, R. (2002). Longitudinal twin study of early reading development in three countries: Preliminary results. Annals of Dyslexia, 52, 49-73.

Dockrell, J. (2009). Causes of delays and difficulties in the production of written text. In R. Beard, J. Riley, D. Myhill, \& M. Nustrand (Eds.), The SAGE handbook of writing development (pp. 489-505). London: SAGE Publications Ltd.

Ellis, A. W. (1984). Reading, writing and dyslexia: A cognitive analysis. London: Lawrence Erlbaum Associates.

Engstrom, E. U. (2005) Reading, writing, and assistive technology: An integrated developmental curriculum for college students. Journal of Adolescent \& Adult Literacy, 49, 30-39.

Goldfus, C., \& Gotesman, E. (20I0). The impact of assistive technologies on the reading outcomes of college students with dyslexia. Educational Technology, 50, 2I-25.

Goldman-Rakic, P. S. (1992). Working memory and the mind. Scientific American, 267, I I0-1 17.

Graham, S., \& Harris, K. R. (200I). Prevention and intervention of writing difficulties for students with learning disabilities. Learning Disabilities Research \& Practice, 16, 74-84.

Gregg, N., Coleman, C., Davis, M., \& Chalk, J. C. (2007). Timed essay writing: Implication for highstakes tests. Journal of Learning Disabilities, 40, 306-3I8.

Helland, T., \& Asbjornsen, A. E. (2010). Digit span in dyslexia: Variations according to language comprehension and mathematics skills. Journal of Clinical and Experimental Neuropsychology, 26, 3|-42.

Hogg, R. V., \& Craig, A. T. (1995). Introduction to Mathematical Statistics. (5th ed.). New York: Macmillan, p. 152.

Jones, D., \& Christensen, C. A. (1999). Relationship between automaticity in handwriting and student's ability to generate written text. Journal of Educational Psychology, 9I, 44-49.

Jordan, I (2002). Visual dyslexia, signs, symptoms and assessment. Scunthorpe: Desktop Publications. www.desktoppublications.co.uk

Kirwan, B., \& Leather, C. (20II). Students' voices: A report of the student view of dyslexia study skills tuition. British Journal of Learning Support, 26, 33-4I.

Lindgrén, S. A., \& Laine, M. (20II). Cognitive-linguistic performances of multilingual university students suspected of dyslexia. Dyslexia, 17, 184-200.

MacArthur, C., Graham, S., Haynes, J., \& De La Paz, S. (1996). Spelling checkers and students with learning disabilities: Performance comparisons and impact on spelling. Journal of Special Education, 30, 35-57.

Menghini, D., Finzi, A., Carlesimo, G. A., \& Vicari, S. (20I I). Working memory impairment in children with developmental dyslexia: Is it just a phonological deficit? Developmental Neuropsychology, 36, 199-213.

Miller-Shaul, S. (2005). The characteristics of young and adult dyslexic readers on reading and reading related cognitive tasks as compared to normal readers. Dyslexia, II, |32-|5|. 
Mortimore, T., \& Crozier, R. W. (2007). Dyslexia and difficulties with study skills in higher education. Studies in Higher Education, 3I, 235-25I.

Olinghouse, N. G., \& Graham, S. (2009). The relationship between the discourse knowledge and the writing performance of elementary-grade students. Journal of Educational Psychology, I0I, 37-50.

Olive, T. (2004). Working memory in writing: Empirical evidence from the dual-task technique. European Psychologist, 9, 32-42.

Olive, T., \& Kellogg, R. T. (2002) Concurrent activation of high- and low-level production processes in written composition. Memory \& Cognition, 30, 594-600.

Olive, T., Alves, R. A., \& Castro, S. L. (2009). Cognitive processed in writing during pause and execution periods. European Journal of Cognitive Psychology, 2I, 758-785.

Pennington, B. F., McCabe, L. L., Smith, S. D., Lefly, D. L., Bookman, M. O., Kimberling, W. J., \& Lubs, H. A. (1986). Spelling errors in adults with a form of familial dyslexia. Child Development, 57, I00I-I0I3.

Peverly, S. T., Ramaswamy, V., \& Brown C. (2007). What predicts skill in lecture note taking? Journal of Educational Psychology, 99, 167-180.

Ramus, F., Rosen, S., Dakin, S. C., Day, B. L., Castellote, J. M., White, S., \& Frith, U. (2003). Theories of developmental dyslexia: Insights from a multiple case study of dyslexic adults. Brain, 126, 84I-865.

Ransdell, S., Levy, C. M. (1996). Working memory constraints on writing quality and fluency. The science of writing: Theories, methods, individual differences, and applications (pp. 93-105). Hillsdale, NJ, England: Lawrence Erlbaum Associates.

Riddick, B. (20I0). Living with dyslexia-The social and emotional consequences of specific learning difficulties/disabilities. London: Routledge.

Siegel, L. S. (1994). Working memory and reading: A life-span perspective. International Journal of Behavioural Development, 17, 109-124.

Smith-Spark, J. H., \& Fisk, J. E. (2007). Working memory functioning in developmental dyslexia. Memory, 15, 34-56.

Snowling M. J., \& Hulme C. (20I I) Evidence-based interventions for reading and language difficulties: Creating a virtuous circle. British Journal of Educational Psychology, 8I, I-23.

Spring, C. (1976). Encoding speed and memory span in dyslexic children. Journal of Special Education, $10,35-40$.

Sterling, C., Farmer, M., Riddick, B., Morgan, S., \& Matthews, C. (1998). Adult dyslexic writing. Dyslexia, 4, I-I5.

Swanson, H. L., \& Ashbaker, M. H. (2000). Working memory, short-term memory, speech rate, word recognition and reading comprehension in learning disabled readers: Does the executive system have a role? Intelligence, $28, \mathrm{I}-30$.

The International Dyslexia Association. (2008). Definition of dyslexia. Retrieved from http://www. interdys.org/ewebeditpro5/upload/Definition.pdf (October 2013).

Vasic, N., Lohn, C., Steinbrink, C., Martin, C., \& Christian, R. (2008). Neural correlates of working memory performances in adolescents and young adults with dyslexia. Neuropsychologia, 46, 640-648.

Wengelin, A. (2007). The word-level focus in text production by adults with reading and writing difficulties. In M. Torrance, L. Van Waes \& D. W. Galbraith (Eds.), Writing and cognition (studies in writing) (pp. 67-82). Elsevier Science: Oxford. 